\title{
Clinical and brain SPECT scan response to zolpidem in patients after brain damage
}

\author{
Nozipho E. Nyakale ${ }^{1}$, Ralf P. Clauss ${ }^{2}$, Wally $\mathrm{Nel}^{3}$, Mike Sathekge ${ }^{1}$ \\ ${ }^{1}$ Nuclear Medicine Department, University of Pretoria, South Africa \\ ${ }^{2}$ Nuclear Medicine Department, Royal Surrey County Hospital, United Kingdom \\ ${ }^{3}$ Pollock Park Medical Centre, Springs, South Africa
}

Correspondence to: Dr. med. Ralf Clauss, MMed (Nuc Med), FRCP, Nuclear Medicine Department, Royal Surrey County Hospital, Guildford, Surrey GU27XX, United Kingdom; e-mail: rclauss@royalsurrey.nhs.uk

\begin{abstract}
Previous reports document transient improvements after daily zolpidem (CAS 82626-48-0) in patients with brain damage. This multi-patient study evaluates the response to zolpidem in neurologically disabled patients, using ${ }^{99 m}$ TcHMPAO brain SPECT scans and clinical rating scales.

Method: 23 of 41 consecutive adult patients, at least 6 months after brain damage were identified as neurologically disabled patients by scoring less than 100/100 on the Barthel Index. Causes of their brain damage included stroke $(n=12)$, traumatic brain injury $(n=7)$, anaphylaxis $(n=2)$, drugs overdose $(n=1)$ and birth injury $(n=1)$. The selected 23 patients had a baseline ${ }^{99 m}$ TcHMPAO brain SPECT scan before starting daily zolpidem therapy and a second within two weeks of therapy, performed $1 \mathrm{~h}$ after $10 \mathrm{mg}$ oral zolpidem. Scans were designated as improved when
\end{abstract}

\begin{abstract}
at least two of three assessors detected improvement after zolpidem. The rest were designated non improved. After four months daily zolpidem therapy, patients were rated on the Tinetti Falls Efficacy Scale (TFES) before and after zolpidem. The TFES ratings were compared using a Wilcoxon non parametric signed rank test. Scan improvers were compared with non improvers, using a two sample t test with unequal variance.

Results: Mean overall improvement after zolpidem on TFES was $11.3 \%$, from $73.4 / 100$ to $62.1 / 100(p=0.0001) .10 / 23$ (43\%) patients improved on SPECT scan after zolpidem. Their mean TFES improvement was $19.4 \%( \pm 16.75)$ compared with $5.08 \%( \pm 5.17)$ in $13 / 23$ non improvers $(p=0.0081)$.

Conclusion: This prospective study adds further evidence to previous reports of zolpidem efficacy in patients with established brain damage.
\end{abstract}

\author{
Key words \\ - CAS 82626-48-0 \\ - Clinical rating scale \\ - SPECT \\ - Stroke \\ - Traumatic brain injury \\ - Zolpidem \\ Arzneimittelforschung \\ 2010;60(4):177-181
}

\section{Introduction}

Zolpidem (CAS 82626-48-0) is a sedative medicine that has been prescribed for insomnia for many years. It induces sleep by activating GABA(A) receptors in the brain which are inhibitory macromolecular complexes that mediate sedative, anticonvulsant, anxiolytic and myorelaxant effects [1]. Zolpidem is a chemically distinct imidazopyridine that binds preferentially to the omega1 subunit of the GABA(A) receptor $[2,3]$.

The medical literature contains several well-documented reports of individual patients with brain damage who improved when they took zolpidem. In 2000 the first case report of an unexpected transient awareness after zolpidem describes a young male patient categorized to the vegetative state after a traumatic brain injury 3 years earlier, who regained consciousness $30 \mathrm{~min}$ after swallowing a $10 \mathrm{mg}$ zolpidem tablet [4]. Several similar cases have been reported since then [58]. Zolpidem has also been reported to improve neurological function in fully conscious patients with severe neurological disabilities due to stroke, traumatic brain injury and other causes [9-12].

In some reports, SPECT and PET scans detected an improvement of suppressed cerebral perfusion and metabolism in the brain after zolpidem $[4,6,11]$. Animal studies have also documented normalization of cerebral perfusion in injured brain after zolpidem [13].

The aim of this study was to evaluate the effect of zolpidem in consecutive patients with neurological disability after brain damage, using established clinical rating scales and ${ }^{99 \mathrm{~m}}$ TcHMPAO brain SPECT scans. 


\section{Patients and methods}

\subsection{Project details}

The study was registered through the Nuclear Medicine Department of the University of Pretoria (UP) as an MMed project of one of the participating co authors (NEN) and approved by the UP ethics committee under project registration number $80 / 2008$.

\subsection{Patient population}

During the last two years, all consecutive adult patients with brain damage of at least 6 months duration who applied to a co author's practice (HWN) for zolpidem therapy were asked if they wanted to participate in the study. All were given full information regarding zolpidem and brain SPECT procedures and ample time to consider the information before they and their relatives gave written consent. All consented patients were fully examined clinically. There were 41 patients who could participate in the study.

\subsection{Patient selection}

To determine neurological disability, all patients were rated according to the Barthel Index after interviews with their carers and families [14]. The top possible score on this index is 100/ 100. 23/41 patients scored less than the possible 100/100. These 23/41 patients were classed as neurologically disabled patients and selected for further study. There were 9 female and 14 male patients, aged between 20 and 77 (mean 45.8). Causes of their brain damage included stroke $(n=12)$, traumatic brain injury $(n=7)$, anaphylaxis $(n=2)$, drugs overdose $(\mathrm{n}=1)$ and birth injury $(\mathrm{n}=1)$. Four were in the Minimally Conscious State, one had the Locked in Syndrome and the rest were Fully Conscious.

\subsection{Clinical rating before and after zolpidem}

Zolpidem tablets (10 mg, Stilnox, Lot 8-64, expiry date July 2012), supplied by Sanofi Aventis South Africa (Pty) Ltd, Midrand, South Africa were provided by the Acadia Pharmacy in Pollock Park, Springs, South Africa. The 23 selected patients took $10 \mathrm{mg}$ zolpidem every morning for at least four months. On follow up, they were scored on the Tinetti Falls Efficacy Scale (TFES) which measures confidence in activities of daily living. The scores were based on interviews with their family and carers who rated the patient's confidence according to the Tinetti criteria at baseline before zolpidem and after zolpidem therapy [15]. Patients and carers were asked whether any sedation was experienced after zolpidem.

\subsection{SPECT scan procedure and preparation}

All selected patients had a baseline ${ }^{99 \mathrm{~m}} \mathrm{TcHMPAO}$ brain SPECT scan before starting zolpidem therapy and a second scan within the first two weeks after starting, performed $1 \mathrm{~h}$ after $10 \mathrm{mg}$ zolpidem, at peak effect. The radiotracer used for the brain SPECT scan was $370 \mathrm{MBq}$ of technetium $99 \mathrm{~m}$ hydroxymethyl propylamine oxime $\left({ }^{99 \mathrm{~m}} \mathrm{TcHMPAO}\right)$. Before the SPECT scans, patients were required to abstain from caffeine or alcohol for $24 \mathrm{~h}$. They were attached to an intravenous infusion line, kept patent by saline and adopted a comfortable position in a dimly lit, quiet room devoid of other people for $30 \mathrm{~min}$. to become as calm and comfortable as possible. A nurse then quietly entered the room and injected the ${ }^{99 \mathrm{~m}} \mathrm{TcHMPAO}$ through the patient's intravenous line.

\subsection{Acquisition protocol}

Scans were conducted at least 30 min after ${ }^{99 \mathrm{~m}}$ TcHMPAO injection in a supine position, using a Siemens Signature series Ecam Dual Head Gamma Camera. The imaging parameters were a $128 \times 128$ matrix, 360 degrees with 3 degree stops, $20 \mathrm{~s}$

Table 1: The gender, age, causes of brain damage (Diagn.: CVA - Stroke, TBI - Traumatic Brain Injury, Anaph - Anaphylaxis, Birth Inj - Birth Injury, Drugs OD - Drugs Overdose), the Barthel Index (BI), the Tinetti Falls Efficacy Scale before zolpidem (TFES), the TFES after zolpidem (TFESzlp), the change in the TFES after zolpidem (change), the state of consciousness (MCS Minimally Conscious State, LIS - Locked in Syndrome, FC - Fully Conscious), brain SPECT scan results (I -Improvers, NI - Non Improvers) and sedation effect (Sed, $y$ - yes, $\mathrm{n}-\mathrm{no}$ ) for 23 neurologically disabled patients (Pt) scoring less than $100 / 100$ on the $\mathrm{BI}$, at least six months after their brain damage.

\begin{tabular}{|c|c|c|c|c|c|c|c|c|c|c|}
\hline Pt & Gender & Age & Diagn. & $\mathrm{BI}$ & TFES & TFESzlp & Change & Consciousness & SPECT & Sed \\
\hline $\begin{array}{l}\text { tl } \\
\text { dm } \\
\text { vj } \\
\text { kd } \\
\text { cl } \\
\text { tk } \\
\text { mn } \\
\text { kc } \\
\text { be } \\
\text { mj } \\
\text { oa } \\
\text { Ip } \\
\text { ra } \\
\text { df } \\
\text { ug } \\
\text { rk } \\
\text { de } \\
\text { Im } \\
\text { he } \\
\text { kt } \\
\text { va } \\
\text { ja } \\
\text { ac }\end{array}$ & $\begin{array}{l}\mathrm{F} \\
\mathrm{M} \\
\mathrm{M} \\
\mathrm{F} \\
\mathrm{F} \\
\mathrm{M} \\
\mathrm{M} \\
\mathrm{M} \\
\mathrm{M} \\
\mathrm{M} \\
\mathrm{F} \\
\mathrm{M} \\
\mathrm{F} \\
\mathrm{M} \\
\mathrm{M} \\
\mathrm{M} \\
\mathrm{M} \\
\mathrm{F} \\
\mathrm{F} \\
\mathrm{M} \\
\mathrm{F} \\
\mathrm{M} \\
\mathrm{F}\end{array}$ & $\begin{array}{l}53 \\
18 \\
61 \\
56 \\
53 \\
65 \\
42 \\
65 \\
22 \\
38 \\
34 \\
53 \\
48 \\
27 \\
39 \\
53 \\
32 \\
72 \\
20 \\
65 \\
77 \\
23 \\
38\end{array}$ & $\begin{array}{c}\text { CVA } \\
\text { TBI } \\
\text { CVA } \\
\text { CVA } \\
\text { CVA } \\
\text { CVA } \\
\text { Anaph } \\
\text { CVA } \\
\text { TBI } \\
\text { CVA } \\
\text { TBI } \\
\text { CVA } \\
\text { Anaph } \\
\text { TBI } \\
\text { TBI } \\
\text { CVA } \\
\text { TBI } \\
\text { CVA } \\
\text { Birth Inj } \\
\text { CVA } \\
\text { CVA } \\
\text { TBI } \\
\text { Drugs OD }\end{array}$ & $\begin{array}{r}45 \\
60 \\
40 \\
20 \\
90 \\
95 \\
60 \\
30 \\
0 \\
95 \\
5 \\
90 \\
10 \\
15 \\
20 \\
80 \\
0 \\
10 \\
45 \\
60 \\
75 \\
80 \\
0\end{array}$ & $\begin{array}{r}73 \\
81 \\
81 \\
100 \\
43 \\
35 \\
86 \\
54 \\
100 \\
36 \\
100 \\
44 \\
100 \\
91 \\
95 \\
71 \\
100 \\
100 \\
66 \\
36 \\
62 \\
34 \\
100\end{array}$ & $\begin{array}{r}25 \\
47 \\
48 \\
71 \\
23 \\
15 \\
70 \\
42 \\
90 \\
26 \\
93 \\
37 \\
95 \\
86 \\
93 \\
69 \\
100 \\
100 \\
66 \\
36 \\
62 \\
34 \\
100\end{array}$ & $\begin{array}{r}48 \\
34 \\
33 \\
29 \\
20 \\
20 \\
16 \\
12 \\
10 \\
10 \\
7 \\
7 \\
5 \\
5 \\
2 \\
2 \\
0 \\
0 \\
0 \\
0 \\
0 \\
0 \\
0\end{array}$ & $\begin{array}{c}\text { FC } \\
\text { FC } \\
\text { FC } \\
\text { MCS } \\
\text { FC } \\
\text { FC } \\
\text { FC } \\
\text { FC } \\
\text { MCS } \\
\text { FC } \\
\text { LIS } \\
\text { FC } \\
\text { MCS } \\
\text { FC } \\
\text { FC } \\
\text { FC } \\
\text { FC } \\
\text { FC } \\
\text { FC } \\
\text { FC } \\
\text { FC } \\
\text { FC } \\
\text { MCS }\end{array}$ & $\begin{array}{c}\text { I } \\
\text { I } \\
\text { I } \\
\text { I } \\
\text { I } \\
\text { I } \\
\text { NI } \\
\text { NI } \\
\text { NI } \\
\text { I } \\
\text { NI } \\
\text { NI } \\
\text { NI } \\
\text { NI } \\
\text { NI } \\
\text { NI } \\
\text { I } \\
\text { NI } \\
\text { NI } \\
\text { I } \\
\text { I } \\
\text { NI } \\
\text { NI }\end{array}$ & $\begin{array}{l}\mathrm{n} \\
\mathrm{n} \\
\mathrm{n} \\
\mathrm{n} \\
\mathrm{y} \\
\mathrm{y} \\
\mathrm{y} \\
\mathrm{n} \\
\mathrm{n} \\
\mathrm{n} \\
\mathrm{y} \\
\mathrm{y} \\
\mathrm{y} \\
\mathrm{y} \\
\mathrm{y} \\
\mathrm{n} \\
\mathrm{n} \\
\mathrm{n} \\
\mathrm{n} \\
\mathrm{y} \\
\mathrm{y} \\
\mathrm{n} \\
\mathrm{y}\end{array}$ \\
\hline
\end{tabular}


per frame and less than $19 \mathrm{~cm}$ radial distance at a $140 \mathrm{keV}$ photo-peak symmetrical $20 \%$ window, using low energy high resolution collimators.

\subsection{Image processing}

Images were reconstructed on an e-soft viewing station using filtered back projection and Metz filtering. Coronal, sagittal and transverse images were constructed with similar alignment in the baseline and post zolpidem images.

\subsection{Interpretation of the brain SPECT scans}

The scans were assessed visually by three independent, experienced nuclear medicine physicians who were unaware of the scan's timing in relation to the zolpidem treatment. Scans were presented to them as within-patient pairs, but in a random order in relation to zolpidem. The assessors were required to state which scan depicted less damage, based on lesion size and uptake, or whether there was no difference. All patients who were rated as better (less damage) on the zolpidem SPECT by two or more assessors were designated improvers while those rated as better on the baseline SPECT or "no different" were designated non improvers. When there was no agreement between assessors they were allowed to reach a consensus opinion while remaining blinded to zolpidem treatment (4/23 patients in total).

\subsection{Statistical analysis}

The statistical analysis was completed by Dr Olorunju from the MRC biostatistics department in Pretoria, SA. The overall TFES score was compared before and after zolpidem in all patients using the non parametric Wilcoxon signed rank test. The TFES scores in patients designated as improved and non improved after zolpidem according to their brain SPECT scans were compared, using a two sample t test with unequal variance.

\section{Results}

\subsection{Patients}

Table 1 shows the gender, age, causes of brain damage, the Barthel Index (BI), the TFES score before and after zolpidem, the change in the TFES score, the state of consciousness (MCS - Minimally Conscious State, LIS Locked in Syndrome, FC - Fully Conscious) the Brain SPECT scan results (I - Improver, NI - Non Improver) and whether there was sedation or not $(y-$ yes, $n-$ no).

Table 2: Mean confidence scores between 1 (most confident) and 10 (least confident) on the Tinetti Falls Efficacy Scale items before and after zolpidem in 23 neurologically disabled patients after brain damage scoring less than $100 / 100$ on the Barthel Index.

\begin{tabular}{l|c|c|c|c}
\hline & $\begin{array}{c}\text { Pre } \\
\text { zolpidem }\end{array}$ & $\begin{array}{c}\text { Post } \\
\text { zolpidem }\end{array}$ & Difference & p value \\
\hline Taking bath or shower & 7.4 & 5.96 & 1.43 & 0.0018 \\
Answering the door or telephone & 6.74 & 5.35 & 1.39 & 0.0049 \\
Walking around the house & 7.48 & 6.17 & 1.3 & 0.0084 \\
Getting in and out of bed & 6.26 & 5 & 1.26 & 0.0085 \\
Getting in and out of a chair & 6.17 & 5 & 1.17 & 0.0145 \\
Reaching into cupboards & 7.82 & 6.7 & 1.13 & 0.0462 \\
Getting dressed or undressed & 7.13 & 6 & 1.13 & 0.0146 \\
Doing simple shopping & 8.26 & 7.26 & 1 & 0.0146 \\
Preparing a meal & 8.17 & 7.21 & 0.96 & 0.0257 \\
Doing light house keeping & 7.91 & 7.39 & 0.52 & ns0.0835 \\
Overall score (all items) & 73.39 & 62.09 & 11.3 & 0.0001 \\
\hline
\end{tabular}

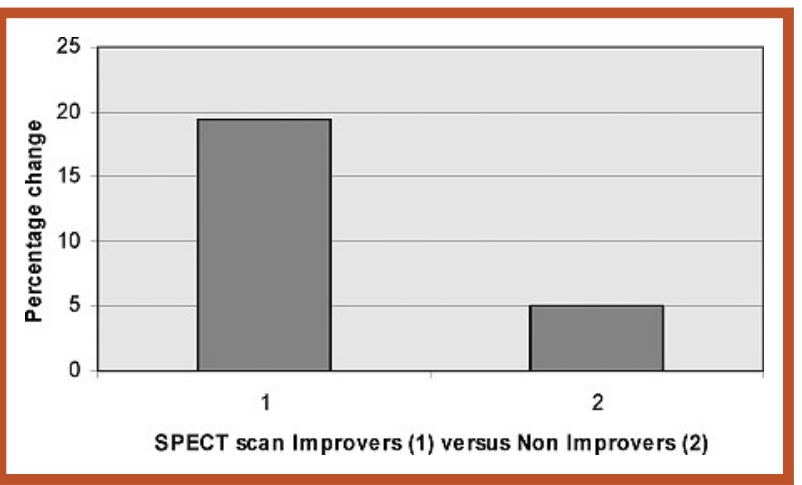

Fig. 1: Mean change on the Tinetti Fall Efficacy Scale after zolpidem in 10 brain SPECT improvers (mean 19.4, SD 16.751) versus 13 non improvers (mean 5.08, SD 5.17).

\subsection{Clinical scale}

Table 2 shows the mean overall score and the mean subsection scores on the TFES before and after zolpidem.

The overall mean improvement after zolpidem on the TFES was $11.3 \%$, a reduction from $73.4 / 100$ to $62.1 / 100$ $(p=0.0001) .10$ out of 23 patients $(43 \%)$ improved on brain SPECT scan after zolpidem while there was no improvement on the SPECT scan in $13 / 23$ patients. In the $10 / 23$ improvers, the mean TFES score fell by $19.4 \%$ $( \pm 16.75)$ compared with $5.08 \%( \pm 5.17)$ in the $13 / 23$ non improvers ( $\mathrm{p}=0.0081$ ) (Fig. 1,2$)$.

\section{Discussion}

This is the first prospective, multi-patient, clinical study to demonstrate the beneficial effect of zolpidem in established brain damage. Our patients' injuries were severe, showing a degree of dependency by scoring less than $100 / 100$ on the Barthel Index (BI). The BI is a widely used clinical tool to measure neurological disability, but is less sensitive for activities of daily living. We chose the equally established, but more sensitive Tinetti scale (TFES) for this purpose. It requires patients or carers to rate confidence in ten activities of daily living on a scale of 1 meaning most confident to 10 meaning least confident. The scale indirectly rates neurological disability because this is linked to lack of confidence in such activities [16]. It also is a measure for the risk of falling, an important consideration in this patient group [5]. We found a highly significant improvement $(\mathrm{p}=0.0001)$ in the mean TFES score after zolpidem in our 23 patients, 10 of whom improved by $10 \%$ or more and 6 by $20 \%$ or more. A $10 \%$ improvement is the equivalent of a patient progressing from inability to perform a task (1/ $10)$ to scoring full marks $(10 / 10)$ which represents a clear and remarkable clinical improvement. 


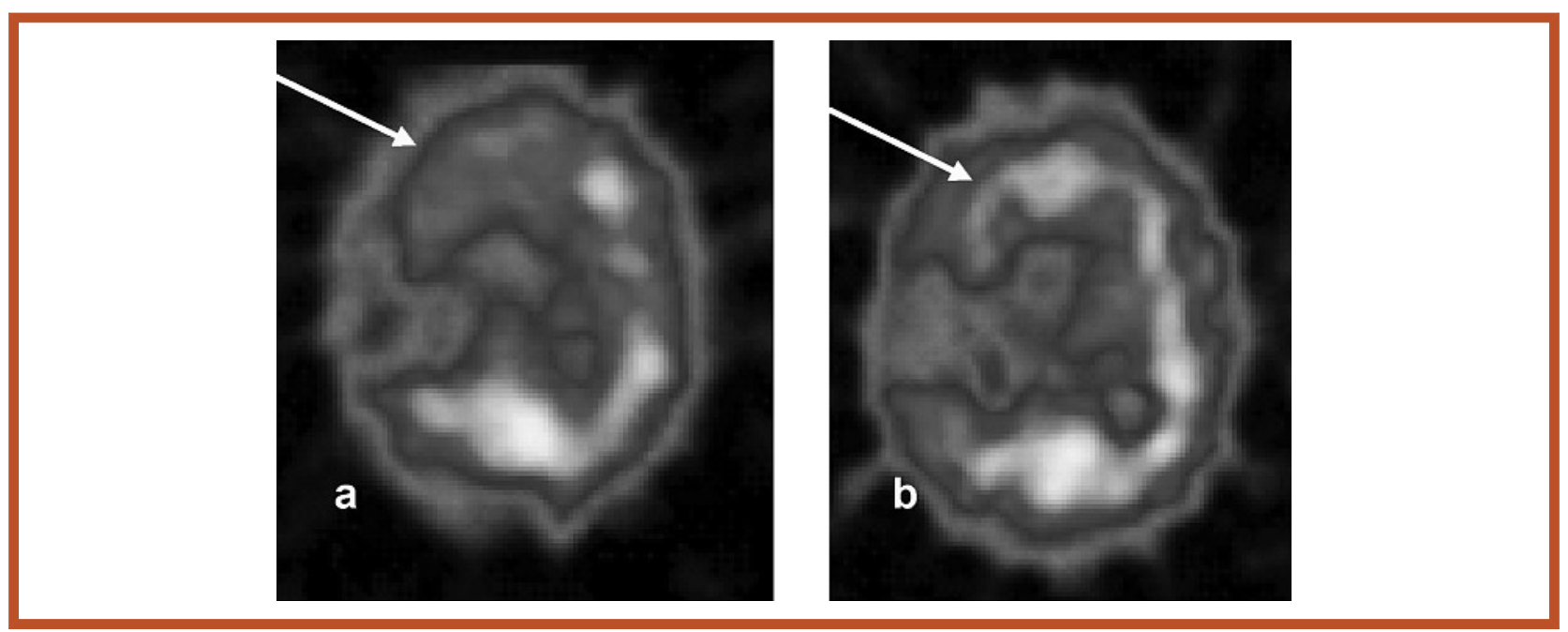

Fig. 2: Example of a transaxial brain SPECT scan slice before (a) and after zolpidem (b) in patient vj. The arrow shows the area of improvement of cerebral perfusion after zolpidem.

There were four patients in the Minimally Conscious State and one patient in the Locked in Syndrome included in this study. The chosen Tinetti Scale may not be the most sensitive way to evaluate these patients, but there are aspects of confidence in such patients which can be well rated on Tinetti criteria, for example "taking a bath or shower, getting in and out of bed and getting in and out of a chair".

We included SPECT scans in this study as objective markers that would not be dependent on clinical rating or sensitive to placebo effects. We found a significant mean TFES improvement in the 10 patients with improved SPECT scans which contrasted with 13 non improvers $(19.4 \%$ versus $5.1 \%, \mathrm{p}=0.0081)$. This indicates a link between clinical and SPECT scan improvements.

While placebo or positive controls are ideal markers for comparative studies, their role in this study appeared questionable due to the obvious sedative effect of zolpidem. In this study 11 of the 23 patients ( $48 \%$ ) reported a sedative effect of zolpidem that would not have occurred on placebo, so rendering a statistical comparison between them invalid. A positive control in the form of another sedative would risk injuries from increased falls because daytime sedation would have been unavoidable for a period of several weeks. In patients who lack confidence for activities like descending steps we considered this to be ethically questionable during a proof of principle study when the beneficial effect of the test treatment remained in question. Having now established that zolpidem has an effect that probably decreases risk to further injury rather than increasing it, our reservations for future trials will be diminished.

We think it improbable that the clinical and SPECT improvements were a placebo effect. Firstly, improvements on the Tinetti Scale were large and clinically more impressive than would be expected from placebos. They were $20 \%$ and over in 6 of the 23 patients which is the equivalent of patients recovering from no confidence whatsoever to full confidence in at least 2 activ- ities. Secondly, all patients were enrolled at least 6 months after their brain injury, in a stable phase, which made spontaneous improvements most unlikely. Thirdly, apart from the objective nature of scans that detect perfusion changes within the brain, we ensured that scans were interpreted blindly and randomly by three independent assessors, who had no knowledge of the clinical rating scores.

We attempted to eliminate selection bias by enrolling consecutively presenting patients regardless of age, gender or cause of injury. This produced a wide variety of injury type and cause, which was not a major concern because previous anecdotal reports have shown beneficial zolpidem effects in a wide spectrum of clinical features and causes. For example the first reported patient was categorized to the Vegetative State after a traumatic brain injury, with a low Glasgow Coma Scale score of 9 that became a normal 15 after zolpidem [4]. By contrast, Hall et al. found improvements after zolpidem in subtle impairments of word recognition and cognitive function in a fully conscious and independent stroke patient [17].

${ }^{99 m}$ TcHMPAO SPECT scans can detect small changes in cerebral perfusion so they are often more sensitive than clinical rating scales. They detect changes regardless of the type of symptom. Improvements after zolpidem have been detected previously in SPECT and PET brain scans, correlating with clinical improvements [46]. The activated areas do not show a typical location or distribution pattern and vary between patients, probably because sites of injury are also highly variable. However, the timing and nature of the changes clearly indicate that they are part of the effect of zolpidem. This was shown pharmacologically in an animal study where the effect of zolpidem activation was blocked by flumazenil, an antagonist at benzodiazepine receptors, which includes the omega-1 sites at which zolpidem acts [18]. We did not find a perfect match between SPECT and TFES improvement, since there were 3 improvers on SPECT scan who had no detectable clinical improve- 
ment on the TFES (patients de, kt and va). This may have been due to a lack of sensitivity to their symptoms in the TFES, which suggests that SPECT scans may be helpful in identifying possible responders when clinical changes are not obvious.

Brain SPECT scans can be interpreted visually by human observers or semi-quantitatively using computerized image comparisons such as BRASS, SPM or VOI techniques [19-21]. However, neither method provides an undisputed gold standard for accuracy and validity since necessary smoothing techniques for example may influence the accuracy of quantitative SPECT techniques [22]. For the unique purpose of this study, namely to identify a response after zolpidem in the same subject, a visual assessment by three independent, blind-to-treatment assessors was thought the most appropriate.

It is unusual that a sedative medication such as zolpidem can produce activation of suppressed brain tissue. It has been postulated that the observed long term physiological brain suppression that occurs after brain injury is a protective GABAergic effect, probably caused by oversensitive modified GABA receptors when GABA reserves become depleted after injury. Zolpidem counters this receptor modification and normalises brain function [5].

In conclusion our 23 patients produced a $43 \%$ response rate to zolpidem therapy according to the brain SPECT scans and clear, significant beneficial clinical effects. Further research should now be undertaken in the form of a placebo controlled trial, to confirm these results in established brain damage, whether it be due to stroke, traumatic brain injury or other causes.

\section{Conflict of interest}

This study was funded by the Nuclear Medicine Department of the University of Pretoria as part of an MMed thesis. No external funding was received for this study. Co authors R. P. Clauss and Wally Nel are honorary consultants to ReGen Therapeutics who are the current holders of the intellectual property on zolpidem use in brain damage.

\section{Acknowledgement}

The authors wish to acknowledge Dr. Andrew Sutton and Dr. Shaun Kilminster for editorial and statistical considerations, the staff of the Nuclear Medicine Department of the University of Pretoria, the MRC statistics department and Mrs. Ilse Myburgh and her team for organising the patient data collection.

\section{Literature}

[1] Swainston Harrison T, Keating GM. Zolpidem: a review of its use in the management of insomnia. CNS Drugs. 2005; 19(1):65-89.

[2] Sanger DJ, Zirkovic B. The discrimination stimulus properties of zolpidem, a novel imidazopyridine hypnotic. Psychopharmacology. 1986;89(3):317-22.

[3] Sanger DJ, Griebel G, Perrault G, Clausne Y, Schoemaker H. Discriminative stimulus effects of drugs acting at GABA(A) receptors: differential profiles and receptor selectivity. Pharmacol Biochem Behav. 1999;64(2):269-73.
[4] Clauss RP, Güldenpfennig WM, Nel HW, Sathekge MM, Venkannagari RR. Extraordinary arousal from semi-comatose state on zolpidem. S Afr Med J. 2000;90(1):68-72.

[5] Clauss RP, Nel HW. Drug induced arousal from permanent vegetative state. NeuroRehabilitation. 2006;21(1):23-8.

[6] Brefel-Courbon C, Payoux P, Ory F, Sommet A, Slaoui T, Raboyeau G, et al. Clinical and imaging evidence of zolpidem effect in hypoxic encephalopathy. Ann Neurol 2007; 62(1):102.

[7] Cohen SI, Duong TT. Increased arousal in a Patient with anoxic brain injury after administration of zolpidem. $\mathrm{Am} \mathrm{J}$ Phys Med Rehabil. 2008;87(3):229-31.

[8] Shames JL, Ring H. Transient reversal of anoxic brain injury-related minimally conscious state after zolpidem administration: a case report. Arch Phys Med Rehabil. 2008; 89:386-8.

[9] Clauss RP, Sathekge MM, Nel HW. Transient improvement of spinocerebellar ataxia with zolpidem. $N$ Engl J Med. 2004;351:511-12.

[10] Clauss RP, Jayarajan V, Nel HW, Saunders E. Evidence for zolpidem efficacy in auditory impairment. 9th European Federation of Neurological Societies Congress 2005; Athens, Greece, September 7-20.

[11] Cohen L, Chaaban B, Haberl MO. Transient improvement of aphasia with zolpidem. N Engl J Med. 2004;350(9):94950.

[12] Shadan FF, Poceta JS, Kline LE. Zolpidem for postanoxic spasticity. Southern Med J. 2004;97(8):791-92.

[13] Clauss RP, Dormehl IC, Oliver DW, Nel HW, Kilian E, Louw WK. Measurement of cerebral perfusion after zolpidem administration in the baboon. Arzneimittelforschung. 2001;51(8):619-22.

[14] Mahoney FI, Barthel DW. Functional evaluation: the Barthel Index. Md State Med J. 1965;14:56-61.

[15] Tinetti ME, Richman D, Powell L. Falls Efficacy as a Measure of Fear of Falling. $J$ Gerontol Psychol Sci. 1990;45(6):239-43.

[16] Bandura A. Self-efficacy mechanism in human agency. Am Psychol. 1982;37:122-47.

[17] Hall SD, Yamawaki N, Fisher AE, Clauss RP, Woodhall GL, Stanford IM. Desynchronization of pathological low-frequency brain activity by the hypnotic drug zolpidem. Nature Precedings 2008; hdl:10101/npre.2008.1966.1

[18] Clauss RP, Dormehl IC, Kilian E, Oliver DW, Nel HW, Louw WKA. Cerebral blood perfusion after treatment with omega receptor drugs, zolpidem and flumazenil in the baboon. Arzneimittelforschung. 2002;52:740-4.

[19] Slomka PJ, Radau PE, Hurwitz G, Dey D. Automated threedimensional quantification of myocardial perfusion and brain SPECT. Comput Med Imaging Graph. 2001;25:15364.

[20] Friston KJ, Holmes AP, Worsley KJ, Poline JB, Frith CD, Frackowiak RSJ. Statistical parametric maps in functional imaging: a general linear approach. Hum Brain Mapp. 1965;2:165-89.

[21] Van Laere K, Koole M, Versijpt J, Dierckx R. Non-uniform versus uniform attenuation correction in brain perfusion SPET of healthy volunteers. Eur J Nucl Med. 2001;28:90-8.

[22] Van Laere K, Warwick J, Versijpt J, Goethals I, Audenaert K, Van Heerden B, et al. Analysis of clinical brain SPECT data based on anatomic standardization and referente to normal data: an ROC-based comparison of visual, semiquantitative and voxel-based methods. J Nucl Med. 2002;43(4): 458-69. 